\title{
Colore delle parole e temperatura dei silenzi nel teatro di Eduardo ${ }^{1}$
}

\author{
Anna Barsotti
}

Università di Pisa

\begin{abstract}
«E chisto è 'o punto», per Eduardo, restituire alle parole il proprio colore, così come alle cose e ai gesti, resistere all'omologazione delle lingue che genera automatismo e imbroglio. Il suo è, dunque, un teatro di contraddizione, che incorpora il variopinto linguaggio della scena per innovarlo dall'interno, agendo da diritto e da rovescio sui prodotti artistici della nuova drammaturgia e della nuova recitazione.

Il fenomeno emerge fin dagli anni Trenta, del mitico «blocco» I De Filippo; anni di sperimentazione laboratoriale, di continuità-discontinuità con la famiglia-compagnia d'origine, da cui prendere le distanze per fondersi nell'organismo della Compagnia Umoristica. Il fratello maggiore più inquieto (e attratto anche da Pirandello) comincia già a sperimentare, negli ambigui "giorni pari», quella tecnica dell'affabulazione e dei silenzi, che diventerà fondamentale nel Teatro di Eduardo con Titina, dopo la traumatica fuga del solista della comicità Peppino. Dalla metà degli anni Quaranta in poi quella tecnica, sostanziando la "trinità eretica" propriamente eduardiana — drammaturgia d'attore, mondo dialettale e teatro in lingua-, diventa l'elemento guida d'una contraddizione scenica diversa, ma sempre più evidente.

Nei «giorni dispari», là dove il rapporto fra individuo e società non si ricostituisce prevalgono nell'arte della recitazione e della commedia del Grande Giuocoliero i monologhi, le pause, l'afasia ovvero il parlare senza le parole; perché nel suo romanzo teatrale, attraversato dal cuneo della comunicazione difficile, non solo dire ma anche non dire è fare. Grazie al colore emotivo delle parole, alla temperatura — calda o fredda — dei silenzi, i problemi del teatro del mondo s'incarnano o, se si preferisce, si denunciano.
\end{abstract}

Parole chiave: Eduardo De Filippo, linguaggio teatrale, teatro di contraddizione.

\section{Abstract}

"And that is the point» for Eduardo — to give the word back its proper colour, the same for things and gestures, to resist any "officialization" of language which only generates automatism and deceit. His, therefore, is a theatre of contradiction, which incorporates the multicoloured language of the scene so that it can be created from within, acting his

1. Il testo della relazione enuclea e rielabora le linee principali d'un saggio nel frattempo già edito: A. BARSOTTI, "La "lingua di contraddizione" nel teatro di Eduardo", in E. TESTONI (a cura di), Eduardo De Filippo, Soveria Mannelli (Catanzaro): Rubbettino, 2005. 
artistic products of new drama and new recitation from the prerogative and from the reverse.

The phenomenon emerges at the end of the 30s, from the mythical «blocco» I De Filippo; the years of workshop experimentation, of continuity-discontinuity with the family-company of origin, from which the basics were embraced to emerge oneself in the Compagnia Umoristica organization. The more restless elder brother (also inspired by Pirandello) already begins to experiment in the ambiguity of "even days», exploring the techniques of the spoken word and silence, which would become fundamental in the Teatro di Eduardo con Titana, after the traumatic fleeing of the comedy front man Peppino. From the middle of the 40s this technique, embodying the Eduardo "heretic trinity" - the drama of the actor, the dialectal world and theatre in language-, becomes the guiding element of a diverse, yet ever more present, scenic contradiction.

In "odd days», where the relationship between the individual and society is not reconstructed, the pauses, the aphasia or rather the speaking without words prevail in the art of recitation and the comedy of the Grande Giuocoliero i monologhi; this because, in his theatrical romance crossed with the wedge of difficult communication, both saying and not saying is doing. Thanks to the emotive colours of the words, to the temperature - hot or coldof the silences, the problems of world theatre are incarnate or, if you prefer, denounce themselves.

Key words: Eduardo De Filippo, Theatrical language, theatre of contradiction.

\section{I}

«E chisto è 'o punto», ${ }^{2}$ per Eduardo, restituire alle parole il proprio colore, così come alle cose e ai gesti, resistere all'omologazione delle lingue che genera automatismo e imbroglio, caos travestito da ordine, bla bla di gente che parla come fosse muta, quando il "pittore» scenico o sociale, non riconosce, impone, è Potere con la p maiuscola.

Da qui l'interpretazione di Dario Fo: «Le parole del potere sono, lo dice Eduardo in una sua poesia, "tutte uguale, e d' o stesso culore: grigio scuro", mentre la parola che scende dal palcoscenico deve essere colorata, star dentro gli umori, i fiati, i ritmi». ${ }^{3}$ E alla fine delle grandi commedie di Eduardo «il sipario calava non sulla fine della vicenda, ma sulla conclusione di un ritmo». ${ }^{4}$

Di fatto, già quelle poesie erano e sono battute, brevi monologhi intercalati da pause; e, quando le leggeva, si susseguivano con marcata semplicità, con una naturalezza inquietante, provocando il vuoto d'ogni altra voce fra gli spettatori risucchiati dalla sua indescrivibile phonè, elaborata sul colore emoti-

2. "Quant'è bello 'o culore d' 'e pparole, / [...] / si chillo c'ha scigliuto / canusceva / 'a faccia, / 'a voce / e ll'uocchie 'e nu tramonto. / Chillo ca sceglie / si nun sceglie buono / se mmescano 'e culore d' 'e pparole. / E che succede? / Na mmescafrancesca / 'e migliar' 'e parole, / tutte eguale / e d' 'o stesso culore: / grigio scuro. / [...] / 'O friddo se cunfonne c' 'o calore / e 'a gente parla cumme fosse muta. / E chisto è 'o punto: / manco nu pittore / pò scegliere 'o culore d' 'e pparole». E. DE FILIPPO, 'E pparole (1971), in Le poesie di Eduardo, Torino: Einaudi, 1976, p. 10-11.

3. D. Fo, Fabulazzo, Milano: Kaos Edizioni, 1992, p. 331.

4. C. Garboli, Casa Cupiello, Roma, 7 maggio 1976, in Id., Un po' prima del piombo. Il teatro in Italia negli anni Settanta, Milano: Sansoni, 1998, p. 229. 
vo delle parole, sulla temperatura — calda o fredda - dei silenzi. ${ }^{5}$ Echi o prove di scena, di quel «romanzo teatrale» (suddiviso poi in Cantata dei giorni pari e dei giorni dispari), nel quale l'artista trinitario, attore-autore e regista, si riserva una parte importante; ne interpreti o meno il protagonista.

L'incipit epigrafico di questo romanzo potrebbe consistere in uno dei più memorabili paradossi eduardiani: "La mia vera casa è il palcoscenico, là so esattamente come muovermi, cosa fare: nella vita sono uno sfollato»; ${ }^{6}$ mentre l'explicit è senza dubbio rappresentato dalla traduzione napoletana del congedo di Prospero nella Tempesta: «Li incanteseme mieje songhe fernute, chellu ppoco de forza ca me rummane è propeta la mia [...]. Mòne da vuje dipenne si aggio da rummanere ccàne cunfinato [...]. Dateme la libertade da li legame mieje cu lu signale de li sbattimane [...]: fiato gentile e nicessario pe' gunfiare li vvele meje, ca si none fallisce lu progetto mio ch'era chillo de fareve divertire». ${ }^{7}$

Un congedo anch'esso aperto, com’è il romanzo delle Cantate, che, anziché determinare un punto d'arrivo, prefigura un punto di partenza per comprendere l'ieri, l'oggi e il domani. Lascia che la memoria personale e collettiva riaffiori — nel «tempo grande»— portando allo scoperto complessi di colpa non rimossi, perché «il teatro muore quando si limita a raccontare fatti accaduti; solo le conseguenze dei fatti accaduti possono raccontare un teatro vivo». ${ }^{8}$

Il romanzo teatrale eduardiano non solo registra, ma sembra presentire gli umori diffusi nella società italiana novecentesca: dagli anni Venti all'apogeo del fascismo, alla seconda guerra mondiale e al secondo dopoguerra, alla restaurazione moderata e all'espansione della società industriale, con le sue successive crisi. Ogni capitolo del romanzo — dove lo spettatore è previsto come «personaggio in più»— rinnova il pensiero teatrale di Eduardo sui problemi della vita, interfacciando «mondo del teatro» e «teatro del mondo»; piano del reale (osservato instancabilmente e persino profetizzato) e quello del fantastico, ancor più capace di pentrare, forse, il ricorrente e basilare "conflitto fra individuo e società». Via via questo romanzo diventa espressione di situazioni artistiche alla ricerca della molteplicità del reale e — nello stesso tempo- di una bruciante e bianca fissione di sogni.

Proprio nelle opere più fantastiche — ispirate dalle fasi di più acuta crisi storica e sociale - sono tanti i segnali politici che illuminano per metafore il desiderio di dialogare con la realtà: da Natale in casa Cupiello a Questi fantasmi!,

5. Ricordiamo, in proposito, lo spettacolo-omaggio che Toni Servillo ha montato sulle poesie di Eduardo, nel 1987, intitolandolo semplicemente "E...". Non Eduardo, punto e a capo, ma "E» puntini puntini. Infatti nello spettacolo (cui ha contribuito anche parte dello staff specializzato di Falso Movimento) le parole si vedevano davvero come colori, e il mare si trasformava in migliaia di mani e di braccia insieme, secondo una poetica in cui l'immagine (corpo, suoni, colori, luce) è valorizzata dalla parola e viceversa.

6. E. DE FILIPPO, Eduardo, polemiche, pensieri, pagine inedite, a cura di I. Quarantotti De Filippo, Milano: Bompiani, 1985, p. 148.

7. E. De FILIPPO, Epilogo (detto da Prospero), in La tempesta di William Shakespeare nella traduzione in napoletano di Eduardo De Filippo, Torino: Einaudi, 1984, p. 182.

8. E. DE FILIPPO, Eduardo, polemiche, pensieri, pagine inedite, cit., p. 151. 
da Le voci di dentro a La grande magia a De Pretore Vincenzo. Appunto perché l'ispirazione e la tecnica teatrali sono per Eduardo inseparabili sia dall'impulso propriamente lirico («molte delle mie commedie» ha detto «sono nate da una poesia») sia dal suo sentirsi partecipe e testimone della società e della storia.

Il filo rosso che collega le diverse prove della sua drammaturgia è quello stesso che attraversa, con il leit-motiv della «crisi del dialogo», la drammaturgia europea del novecento, da Pirandello a Ionesco a Beckett... D'altra parte la sua è una drammaturgia d'attore, e d'attore di origine dialettale, la cui lingua consiste anche nella fisicità dei corpi, all'interno di un sistema dove il rapporto fra la sala e il palco è fondamentale e immediato. La parola eduardiana è una parola agita, il cui luogo naturale è la scena; perciò è predisposta non al «sempreuguale», ${ }^{9}$ ma a conversazioni teatrali sempre nuove, senza confini, fra diversi interpreti e diverso pubblico.

La sua è quasi sempre una drammaturgia della comunicazione difficile, ma in cui dire è fare: ne deriva l'oscillazione costante fra la rappresentazione dell'individuo isolato in un mondo che non lo capisce e la resa dei suoi tentativi di costruire un rapporto di comunicazione con gli altri. Altri come personaggi, altri come spettatori: perché in quella compagnia di personaggi-uomo, buffoneschi e melodrammatici, ironici e minacciosi, in un angolino della foto di gruppo... ci siamo sempre anche noi!

Nato esattamente all'inizio del '900, Eduardo testimonia col suo teatro lo svolgimento di un'epoca che è ancora sostanzialmente la nostra; è difficile immaginare un repertorio più ricco e più sensibile del suo, per chi vorrà osservare in futuro quel «secolo difficile». Il suo modo di rappresentare storie ha molto dell'atto del ricordare: storie di gente che ha vissuto, amato, sofferto, riso, deriso ed è stata derisa; ha provato gli inverni della guerra e le primavere (effimere) della pace, trovato sia negli uni che nelle altre "giorni pari» e "giorni dispari». Perché la "gente» eduardiana, che ha guardato il mondo con l'aiuto o nel disorientamento di sempre più perfezionate «scoperte» (ricordate il Sindaco del rione Sanità? "la Radio, la televisione, l'atomica, lo Spuntnik...»), non ha mai perso di vista il suo punto di riferimento essenziale, una famiglia (naturale o teatrale), una Città...

Ma proprio la sua cultura d'attore e d'attore napoletano, nato e cresciuto in un ambiente "differente», di forte resistenza della tradizione ad ogni prassi d'omologazione scenica, ha guidato Eduardo nella creazione di una drammaturgia che a partire dal linguaggio — l' «eresia dialettale» - stabilisce rapporti controllati fra le storie da raccontare e quelle da mostrare al pubblico, bloccando il naturalismo dei comportamenti esibiti, frazionando i meccanismi abituali del discorso, e aprendo vuoti, pause, silenzi attivi attraverso cui evocare profondità poetiche: tensioni negate, bisogni nascosti, paure inconsapevoli.

9. M. GRANDE, «Datemi un attore», Rinascita, n. 37, 22 marzo 1984, p. 21. 
«I suoi silenzi erano la testimonianza del suo essere parsimonioso con le parole», scrive Fo, «perché sapeva che le parole hanno un peso». ${ }^{10}$ Nel teatro di Eduardo le parole hanno ancora un senso, possono essere usate come armi di offesa e di difesa, anche quando s'imbrogliano o vengono a mancare: grazie alla matrice partenopea dell'attore-che-scrive, al suo bagaglio di abitudini generative d'un repertorio che contamina livelli e generi diversi di spettacolo. Da quel teatro della memoria hanno continuato ad estrarre brandelli d'abiti e tracce di trucco Santanelli, Ruccello, Moscato e gli altri: come la linea petitiana e scarpettiana ha potuto ricollegarsi - tramite Eduardo- a un linea pirandelliana, oggi, le opere dei nuovi drammaturghi napoletani —attraverso Pinter, Genet, Beckett e Artaud - possono ricollegarsi a Viviani e a Eduardo. ${ }^{11}$

Napoli è un "paese curioso", recita Eduardo: «un teatro antico, sempre apierto. / Ce nasce gente ca, senza cuncierto, / scenne p' 'e strate e sape recità». ${ }^{12}$

Napoli ha, sul piano antropologico, un comportamento sociale recitante: questo è al fondo di una tradizione scenica che oltrepassa Scarpetta padre, il suo diverso maestro Petito, e risale alla Commedia dell'Arte. Il nostro attoreautore coglie l'innata disponibilità performativa dei suoi personaggi, «li prende e li mette sul palcoscenico», ne ride, senza deriderli mai del tutto perché, allo stesso tempo, «li tortura» e li compatisce. Può mescolare il comico al tragico, e proprio questa confusione partorisce, per Servillo, «la lingua teatrale più straordinaria, la felicità drammaturgica più straordinaria che c'è in questo paese e che non c'era da Goldoni». ${ }^{13}$

L'ascendenza artistica di Eduardo spiega anche il suo rifiuto d'ogni manifesto, delle stessa parola impegno: «Scrivere una commedia impegnata è facile», egli osserva, «il difficile è impegnare il pubblico ad ascoltarla». ${ }^{14} \mathrm{E}$ dal modo in cui rivive il teatro di tradizione, fatto di conflittualità e di riconoscimenti, discende quel suo speciale distacco investigativo —ora scaldato ora raffreddato da un'equivalente comicità - nella raffigurazione di personaggi e ambienti. "Occhi e orecchie miei sono stati asserviti da sempre», dice, «a uno spirito di osservazione instancabile, ossessivo che mi ha tenuto $[\ldots]$ inchiodato al mio prossimo e che mi porta a lasciarmi affascinare dal modo d'essere e di esprimersi dell'umanità». ${ }^{15}$ Tale distacco e l'alone comico o d'ironia grottesca, spesso sofferta, sono serviti al detective-creatore di storie per proiettare e graduare sulla scena «i conflitti interiori e interpersonali, il contrasto fra individui disarmati e la forza del potere (politico, economico, ideologico che sia)». ${ }^{16}$

10. D. Fo, Fabulazzo, cit., p. 43-59.

11. Cfr. F. TaViani, "Eduardo e dopo», in «Dossier: Eduardo De Filippo e la sua eredità", a cura dello stesso, Lettera dall'Italia, n. 19, 1990, p. 21-40, p. 24.

12. E. DE FILIPPO, Baccalà (1949), in Le poesie di Eduardo, cit., p. 191.

13. T. Servillo, Intervista da me condotta presso il Teatro Verdi di Pisa, il 28 febbraio 2004, in occasione d'una ripresa di Sabato, domenica e lunedi.

14. E. DE FILIPPO, Eduardo, polemiche, pensieri, pagine inedite, cit., p. 164.

15. E. De Filippo, Nota, a I Capolavori di Eduardo, Torino: Einaudi, 1973, vol. I, p. VII.

16. L. VILLARI, «Eduardo: il senso e la malinconia della storia», Nuovi Argomenti, n. 15, 1985, p. 4. 


\section{III}

Dario Fo ha detto, ai funerali di Eduardo: «nel suo modo di usare l'arma dell'ironia come strumento di denuncia dell'ingiustizia, non c'erano né rassegnazione né rinuncia [...] adorava il sarcasmo, anche nelle scene più addolorate, patetiche, non poteva fare a meno di piantarci lo sganascio dell'ironia». ${ }^{17}$ Il «giullare del popolo» riconosce al vecchio «giuocoliero» anche l'invenzione di un «lessico completamente nuovo, un linguaggio con il quale sapeva farsi capire da tutti». Proprio per questo, aggiunge, il suo teatro «non ha mai parlato il linguaggio del potere»; addirittura, "prendendo di mira il potere, il primo obiettivo di Eduardo è stato il linguaggio». ${ }^{18}$ Eppure se entrambi hanno in comune l'aspirazione a produrre coscienza negli spettatori mediante il riso e il paradosso, il più vecchio ha teso, piuttosto che a provocare beffardamente il pubblico, a colpevolizzarlo senza darlo a vedere, senza prenderlo di petto.

Invece di infilargli «il dito nell'occhio», ha preferito aprirglielo, l'occhio, e magari tutti e due, socchiudendo sornione uno dei suoi. "Io non credo nel tagliare le teste ma nel cercare di farle pensare», ha detto ad un gruppo di studenti nel 1976; ${ }^{19}$ ma in un suo aforisma malizioso: «quando il pubblico ride troppo a teatro, non pensa mai di aver riso di se stesso, e forse perciò dà del buffone all'attore che è riuscito a farlo ridere troppo». ${ }^{20} \mathrm{Il}$ comico (che anche per lui, come per Fo, nasce dal tragico) diventa strumento della sua superiore malizia nel porgere argomenti spinosi: per far sì che il pubblico al quale materiali tanto colpevolizzanti vengono offerti, sviato o tranquillizzato da quell'umorismo, li accolga e "ci ragioni».

In tal modo, il conflitto continuo rappresentato dal suo teatro - fra l'Io e il Mondo, l'individuo e la società o il Potere-, provoca nello spettatore una sensazione di disagio, di "disparità», anche nei "giorni pari», e produce l'avvertimento risentito di assurde discrepanze fra la storia minuscola e la storia maiuscola. Il protagonista eduardiano va controcorrente: intraprende un viaggio periglioso e inattuale attraverso la Storia, quando essa ha perduto ogni privilegio di consequenzialità (o almeno così pare). È uno spostato ma anche uno "scampato», che da "ectoplasma» aspira a ridiventare "uomo». E il viatico che gli occorre, in questo percorso verso un nuovo umanesimo, è la "parola adatta», la "parola colorata»che possa riattivare quel dialogo entrato in crisi nella cosiddetta realtà, come nel suo doppio, il teatro.

«Teatro significa vivere sul serio quello che gli altri, nella vita, recitano male»: ${ }^{21}$ Eduardo credeva nel valore di scambio fra teatro e vita sociale. Si era posto l'obiettivo di percorrere «la sottile e difficile linea di confine tra l'invenzione, la fantasia e la vita» immaginando di attraversare questa linea nei due

17. D. Fo, «Testimonianze» (agosto 1986), in Eduardo De Filippo. Vita e opere 1900-1984, a cura di I. Q. De Filippo e S. Martin, Milano: Mondadori, 1986, p. 189.

18. D. Fo, Fabulazzo, cit., p. 331.

19. E. DE FILIPPO, Eduardo, polemiche, pensieri, pagine inedite, cit., p. 176.

20. Ivi, p. 148.

21. E. De FILIPPO, cit. da L. Villari, Eduardo: il senso e la malinconia della storia, cit., p. 4. 
sensi e di regolare la realtà con «l'orologio della fantasia». ${ }^{22}$ "Nu relogio cumpiacente» - scrive in un'altra poesia — "ma che batte con il ritmo giusto, che fa "tà-tì, tà-tì, nun fa tì-tà" ". ${ }^{23}$

Il suo è, dunque, un teatro di contraddizione, che incorpora (anzi ha nel DNA napoletano) il variopinto linguaggio della scena per innovarlo dall'interno, agendo da diritto o da rovescio anche sui prodotti artistici della nuova drammaturgia e della nuova recitazione, certamente non solo partenopee. $\mathrm{Ci}$ sono gli eredi diretti, naturalmente, come il figlio Luca, ma fra gli indiretti possiamo citare Bene, Leo de Berardinis, Cecchi, oltre allo stesso Fo.

\section{IV}

Il fenomeno della contraddizione emerge fin dagli anni Trenta, quelli mitici del «blocco» I De Filippo, con i grandi fratelli Titina e Peppino; anni di sperimentazione laboratoriale, di continuità-discontinuità con la famiglia-compagnia d'origine (di Scapetta padre e di Scarpetta figlio), da cui bisogna prendere le distanze per fondersi in un nuovo organismo artistico, la «Compagnia Umoristica», per un decennio almeno, d'eccezione. Sono gli anni dell'eresia dialettale, in cui il «sillabario» ereditato da padre-zio Scarpetta, combinandosi a seconda delle situazioni e dei personaggi, diventa senza provocazioni apparenti un contraltare all' "antilingua recitativa» ${ }^{24}$ che tendeva ad uniformare, attraverso il "grigio scuro» imposto dalla cultura dominante, il panorama dei nostri palcoscenici. "Erano gli anni in cui il regime fascista perseguiva una politica antidialettale e xenofoba che [...] condizionava il mondo del teatro con il mancato finanziamento delle compagnie dialettali». ${ }^{25} \mathrm{Ma}$ la lengua napoletana dei De Filippo può prendersi gioco di direttive e veline ministeriali grazie ai trucchi del mestiere, proprio perché la tradizione in cui si radica è quella della lingua dei corpi, delle parole, dei gesti e degli oggetti che sul palco vivono, incontrando il gradimento non solo del pubblico ma persino di qualche (non si sa se volutamente) distratto personaggio del potere.

Uomo e galantuomo, Ditegli sempre di sì e Sik-Sik, l'artefice magico sono esemplari di tale alibi umoristico: ogni «situazione» è risucchiata da un "concertato» che costruisce un sistema di bugie (o di illusioni) rischiarate da verità folgoranti, ma dove l'ultima verità, di volta in volta, denuncia la prepotenza del più forte. Il capocomico Gennaro De Sia è costretto a fingersi pazzo per non pagare i debiti; Michele Murri ritorna in manicomio; perfino l'illusionista-illuso Sik-Sik piange nascostamente l'esito fallimentare dei propri giochi

22. L. VILLARI, Ibidem.

23. E. De Filippo, Fantasia, in Le poesie di Eduardo, cit., p. 73.

24. C. Meldolesi, «Gesti parole cose dialettali. Su Eduardo Cecchi e il teatro della differenza», Quaderni di Teatro, VIII, n. 31, 1986, p. 141; si veda anche di MELDOLESI, La trinità di Eduardo: scrittura d'attore, mondo dialettale e teatro nazionale, in Fra Totò e Gadda. Sei invenzioni sprecate del teatro italiano, Roma: Bulzoni, 1987.

25. N. De BlasI, «Uno scrittore tra dialetto e italiano», in E. DE FiLIPPO, Teatro, vol. I, Cantata dei giorni pari, a cura di N. De Blasi e P. Quarenghi, Milano: Mondadori, 2000, p. LVI. 
di prestigio. Per non parlare di Natale in casa Cupiello, che come tutte le grandi farse (il Borghese gentiluomo o il Malato immaginario) non aveva "fine", in origine. Ma attraverso la sua singolare biografia artistica — tre atti scritti e recitati in anni diversi, dal '31 fino alla loro prima edizione omogenea nel '43 - getta un ponte mobile verso la produzione della maturità, quella dei giorni (soffertamente) «dispari». Quel rissoso interno di vita napoletana denunciava la miseria dell'intero paese proprio nei "giorni pari», ed appariva già costruito per simboli e per lapsus sapienti. L'intreccio (la storia di un adulterio in famiglia) era continuamente marginalizzato dalla vita che ammicca ed esplode improvvisa: fra i lazzi di Peppino-Nennillo, i sussurri, le grida e i deliqui di TitinaConcetta, emergeva «la corrucciata, fantastica, disappartenenza alla vita di Eduardo-Lucariello [...], vittima di un incantesimo che non è solo la fabbrica di un presepio». ${ }^{26}$

D'altra parte, gli anni Trenta e i primi anni Quaranta sono anche quelli delle traduzioni e ricreazioni dalle opere del secondo padre-maestro eduardiano, Luigi Pirandello, della sperimentazione parallela o alternata di una lingua più vicina a quella nazionale: dove il gioco con il Potere è più subdolo, dal momento che l'italiano parlato da certi personaggi borghesi (Uno coi capelli bianchi, Io, l'erede) non è mai l'antilingua teatrale del «fare pensoso» e della «disinvoltura», 27 ma, già mescolandosi al dialetto, trova suoni disarmonici rispetto al coro, al concerto istituzionale. Non a caso, ricorda Savinio, «i commendatori e le loro signore venuti a farsi quattro risate, ridono sì, ma con la bocca storta» (Omnibus, 19 novembre 1938).

Di fatto Eduardo — come riafferma Fo — «si è sempre rifiutato di parlare in "italianesco" o "italiota", cioè nella lingua semi ufficiale, burocratica, libresca, radiofonico-televisiva del regime» ${ }^{28}$ Da parte sua Eduardo osservava: «a Napoli sono passati in tanti e per capirci ci si intendeva a gesti. Poi si inventavano le parole. E così è stato nel teatro». In questa battuta, l'origine della sua personale napoletanità: non come «cosa immobile» ma come «linguaggio che si evolve a seconda dei periodi storici». ${ }^{29}$

Egli incomincia anche a sperimentare, negli ambigui «giorni pari» —che produranno via via disagio e incrinature nel blocco dei De Filippo- quella tecnica dell'affabulazione e dei silenzi, che diventerà fondamentale nel Teatro di Eduardo con Titina, dopo la traumatica fuga del solista della comicità Peppino nel 1944. Dalla metà degli anni Quaranta in poi quella tecnica, sostanziando la trinità eretica propriamente eduardiana —drammaturgia d'attore,

26. C. Garboli, Casa Cupiello, cit., p. 228.

27. C. MeldOleSI, «Gesti parole e cose dialettali. Su Eduardo Cecchi e il teatro della differenza», Quaderni di Teatro, n. 12, 1981, p. 132.

28. D. Fo, Fabulazzo, cit., p. 331.

29. E. De Filippo, cit. in M. NAVA, «La Napoli dei "giorni dispari”", Corriere della Sera, 17 gennaio 1983. 
mondo dialettale e teatro in lingua-, diventa l'elemento guida d'una contraddizione scenica diversa, ma sempre più evidente.

Nel corso dei giorni dispari, là dove non si ricostruisce il rapporto fra individuo e società (come la sospensione finale di Napoli milionaria! aveva lasciato sperare), prevalgono i monologhi, le pause, l'afasia ovvero il parlare senza le parole nell'arte della recitazione e della commedia del Grande Giuocoliero; perché nel suo teatro non solo dire, ma anche non dire è fare.

Eppure, fin dagli anni Trenta Vittorio Viviani scrive: «Quando tace Eduardo è un perfetto oratore»; ${ }^{30}$ e Silvio d'Amico, per una ripresa di Uomo e galantuomo nel '49, osserva come Eduardo interprete di Gennaro De Sia, un "professionista della finzione», ${ }^{31}$ muovesse dalle apparenze di un linguaggio franto, incespicato e ribattuto, tale da dare all'ingenuo l'impressione della più ovvia quotidianità, ma per arrivare a ben altro...

Non a caso spettatori e artisti tutt'altro che ingenui, come Pasolini e Garboli, Flaiano, Savinio, Fo, Cecchi insistono sulla differenza della lingua in scena di Eduardo rispetto alla condizione normale del «recitare all'italiana»; a quello stereotipo dell' «attore di voce» che aggiornava un compromesso stretto in tempi lontani tra «la lingua media scritta e le condizioni sceniche borghesi». ${ }^{32}$ Negli anni sessanta, Pasolini afferma che il suo è in realtà un italiano orale parlato dai napoletani, ma non ne fa una mimesi naturalistica: vi ha creato sopra una convenzione che gli dà assolutezza, è una purissima lingua teatrale. ${ }^{33} \mathrm{E}$ Cecchi confiderà come sulla propria ricerca abbiano influito, negli stessi anni, «da una parte il grande teatro napoletano, Eduardo, e dall'altra [...] il Living Theatre». ${ }^{34}$

Per cancellare il malinteso del naturalismo di Eduardo, bisogna osservare i nessi tra l'origine dei suoi singolari primi piani, la straordinaria capacità di parlare senza le parole, e la ricorrente alternanza di affabulazione e silenzi nell'ambito della sua stessa drammaturgia d'attore. La sua è una naturalezza inquietante proprio perché costruita, basata su una costante e mirata dialettica tra il volto e la maschera. L'attore-autore creava con il personaggio un rapporto alternato di distacco e partecipazione, un gioco che coinvolgeva il rapporto stesso del pubblico col personaggio, e consentiva allo spettatore di ridere di quel personaggio e, al tempo stesso, di partecipare al suo dramma. Quel gioco dell'attore si trasforma immancabilmente, nel passaggio dalla scena alla pagina e viceversa, in arte della commedia.

30. VIVIANI, «Eduardo De Filippo», Il Mattino illustrato, XIX, n. 35, 1936; ora in Id., Storia del teatro napoletano, Napoli: Guida, 1969, p. 901.

31. S. D’Amico, «Eduardo lirico del dialetto», Il Tempo, 23 dicembre 1949.

32. C. Meldolesi, «Gesti parole e cose dialettali. Su Eduardo Cecchi e il teatro della differenza", cit., p. 132. cfr. anche Id., La trinità di Eduardo, cit.

33. Rispondendo all'inchiesta su Gli scrittori e il teatro, curata da M. Rusconi, in Sipario, XX, n. 229, 1965.

34. C. CECCHI, «Palermo e una trilogia», Patalogo ventuno, annuario dello spettacolo 1998, Torino: Ubulibri, 1998, p. 184; dirà anche che "Eduardo è in sé un accadimento teatrale [...] andava in scena, e subito succedeva qualcosa di grandissimo.» (Cecchi cit. in G. DEL RE, «Recito, rischio, taglio il monologo», Il Messaggero, 12 luglio 1912). 


\section{VI}

A quali espedienti ricorre Eduardo per rendere spettacolare la crisi del dialogo? Il suo protagonista incomincia con l'inventarsi un linguaggio privato, come l'artefice magico Sik-Sik, che s'illude e cerca d'illudere il «rispettabile pubblico", contaminando parlata regionale e linguaggio elevato, ma s'aggroviglia nei labirinti della grammatica: "Qui non s’imbroglia a nisciuno. Questi sono tutti giuoca senza priparato». ${ }^{35}$ È già un anti-linguaggio che si condensa in formule ripetute caparbiamente, con effetti comici stravolgenti, finché il messaggio non venga ricevuto da chi ascolta. Questa terapia della parola, che si sforza d'infrangere la solitudine propria e l'incredulità altrui, diventa impraticabile per il protagonista di Ditegli sempre di sì.

"C’è la parola adatta, perché non la dobbiamo usare», insiste maniacalmente Michele Murri; eppure la formula magica del pazzo/savio smonta i giochetti di vanità, ipocrisia o egoismo che i cosiddetti normali alimentano attraverso l'uso "grigio scuro» delle parole. Quanto a Luca Cupiello, non riesce a riunire neppure con la "difficile parola» la sua ideale, presepiale, famiglia-società; anche lui Don Chisciotte e artista incompreso, isolato in mezzo ai suoi perfino nel linguaggio: «Niente!... È un parlare speciale»; «Niente, niente... è un altro linguaggio!». ${ }^{36}$ Esemplare, al limite, il monologo sul «fatto dei fagiuoli» nell'epilogo del Natale: un antilinguaggio ben al di là della giustificazione naturalistica, la paresi; si tratta d'un grammelot napoletano.

Prima di essere un attore dialettale, Eduardo è un autore della «verità esclusa", che esibisce il trapasso dal non rappresentabile (il dramma messo in crisi dalla comunicazione difficile) al rappresentabile (la spettacolarizzazione di tale crisi) giocando sugli ambigui rapporti fra lingua e dialetto, come tra affabulazione e silenzi. Accadrà a Gennaro Jovine, comiziante e finto morto, a Pasquale Lojacono, pantomimo e dialogante con interlocutori (e spettatori) fantasma, ad Alberto Stigliano, finto muto e riconquistatore della parola, a Guglielmo Speranza, io epico borghese e figurino da varietà, risorto proprio nell'occasione grottesca dei suoi funerali. Creature di un attore che scrive, e che con la sua parte intrattiene rapporti di distacco e di partecipazione.

La tecnica dei leit-motive ostinatamente emessi dalla voce discorde, fuori dal coro, diventa funzionale per Gennaro Jovine («'A guerra nun è fernuta... e non è fernuto niente!»), ma solo nella Napoli milionaria! del '45. D'altra parte con quella "commedia storica" comincia nel 1951 la pubblicazione del romanzo teatrale eduardiano. La Cantata dei giorni dispari (che precede editorialmente la Pari) è contraddistinta da una corrente alternata lingua-dialetto, con ogni possibile interferenza fra i due registri; si attiva la vitalità dialettale in promiscuità con il teatro in lingua, sovvertendo le distinzioni regolamentari.

35. E. De FILIPpo, Sik-Sik, l'artefice magico, in Id., Cantata dei giorni pari, cit., p. 231-232.

36. La prima versione della battuta è in E. DE FILIPPO, Natale in casa Cupiello, in Id., Cantata dei giorni pari, Torino: Einaudi, 1959, atto I, p. 233; la seconda costituisce una delle varianti introdotte dall'autore nel testo, risale all'edizione Einaudi del 1982, e si trova ora nella Cantata, cit., p. 371. 
E, al di là dei copioni originari, nel testo «consuntivo» di Napoli milionaria! il dialetto appare più legato all'espressione dei sentimenti o del "recitar parlando» che alla resa naturalistica di un ceto sociale.

D'altra parte, la tendenza al monologo dei protagonisti eduardiani apre canali obliqui di comunicazione con gli spettatori. Ma il passo dal monologo all'afasia è breve, ed evidenzia il rifiuto di quei rapporti che, nel dialogo convenzionale, sono divenuti finzione. Perciò la difficoltà a parlare insieme risalta nelle commedie che testimoniano o prefigurano cambiamenti critici della società: Questi fantasmi! (1946), Le voci di dentro (1948), Mia famiglia (1955). Si passa dal dialogo-monologo di Pasquale Lojacono con un "personaggio in più» trasparente al codice pirotecnico dello "sparavierze» $\mathrm{Zi}$ " Nicola, fino a giungere al mutismo, ancora strumentale, di Alberto Stigliano. Se il conformismo è un'arma del potere in grado di condizionare, con le sue parole grigio scuro, qualunque creatività e spontaneità dell'individuo, la sfasatura dei linguaggi doveva per forza sconfinare nella soluzione del silenzio.

Lespediente del monologo dialogato arriva al punto di fondare il senso d'una commedia tutta giocata sull'ambiguità, come Questi fantasmi!' Nella parte del professor Santanna, anima utile ma che non compare mai, sono appunto gli spettatori, ossia «l'occhio del mondo delegato a un dirimpettaio»: lo dice Eduardo a Montalcino nell' '83. E nell'edizione televisiva del '62 il monologodialogo finale ripristina l'ambiguità dell'opera, soprattutto attraverso l'interpretazione del protagonista. Pasquale-Eduardo usa in modo contrastivo il linguaggio fonico e quello gestuale: «Professo', professo', avevate ragione voi... I fantasmi esistono [...] Ci ho parlato... Mi ha lasciato una somma di danaro... (Mostra i biglietti) Guardate... Però dice che ha sciolto la sua condanna, che non comparirà mai più... (Ascolta) Come?... Sotto altre sembianze? Ė probabile... E speriamo...». ${ }^{37}$ Mentre pronuncia la battuta "E speriamo...»" quasi con candore, esibisce e conta freneticamente i bigliettoni!

Quanto ai silenzi, pensiamo a Mia famiglia: Eduardo nasconde abilmente al pubblico la chiave della sua parabola, solo a metà del secondo atto sappiamo che l'afasia del pater familias, Alberto Stigliano, non dipende da una patologia ma dalla sua convinzione dell'inutilità delle parole. E appunto perciò, nelle scene senza parole, il creatore dello spettacolo realizza strategie coreografiche fra i gruppi e il singolo (condensate nelle didascalie) ed evidenzia i mezzi rappresentativi attraverso lo stoppare, l'accelerare o il rallentare. Il silenzio del protagonista diventa una presenza perché è uno dei punti in cui il gioco del teatro passa attraverso la sua sospensione, provocando sia gli altri attori sia il pubblico. Anche il silenzio fra una battuta e l'altra, o fra una parola e l'altra, caratterizza la scrittura scenica eduardiana, ma anziché provocare distrazione attira l'attenzione, suscitando disagio (con se stessi) e curiosità. Più che pause, piccole voragini, strappi talvolta ricuciti da ripetizioni in italiano o in dialetto (a seconda dei casi) della parola che ha preceduto quel silenzio. Allora la sospen-

37. E. De FILIPPO, Questi fantasmi!, in Id., Cantata dei giorni dispari, a cura di A. Barsotti, Torino: Einaudi, 1998, vol. I, atto III, p. 181-182. 
sione consente anche i passaggi da un piano di lettura all'altro (della situazione e del personaggio) e magari la loro dialettizzazione progressiva, nel senso della differenza linguistica come forma di «epicità». Ma in Eduardo è la tradizione comica e farsesca o del varietà ad agire dal profondo, magari cambiando magicamente di segno, dal comico al tragico, senza dover passare al filtro di Mejerchol'd e della bio-meccanica, eppure operando in sintonia con il gusto dell'avanguardia europea (Majakowskij, Brecht).

\section{VII}

Il maggiore contrasto tra la sua arte affabulatoria e quella del silenzio si concentra in Gli esami non finiscono mai, ma il fenomeno appare intrigante in una commedia che, invece di alternare nello stesso personaggio affabulazione e silen$\mathrm{zi}$, scinde le due funzioni fondamentali in due diversi, corrispettivi, personaggi. $\grave{\mathrm{E}}$, in modo significativo, una commedia sulla phoné, Le voci di dentro, "Tarantella» in tre atti. Di dentro escono le voci mulinanti nella testa di Alberto Saporito, nuovo Don Chisciotte eduardiano che prima sfoga la propria confusione coscienziale denunciando i vicini, e alla fine, scoperta la causa della sua ed altrui mostruosità («in mezzo a voi, forse, ci sono anch'io, e non me ne rendo conto»), ${ }^{38}$ trasforma quell'accusa gridata con enfasi dialettale in un'arringa davvero "precisa e documentabile».

«Mo se sono imbrogliate le lingue», dice all'inizio il portiere Michele, «Ecco che la notte ti fai la fetenzìa di sogni»; 39 e, appunto perché le voci di dentro non corrispondono più alle voci di fuori, a forza di reticenze, sospetti reciproci e ipocrisie si può arrivare a mettere «un assassinio [...] nelle cose normali di tutti i giorni ... ", come nella denuncia finale del protagonista: "Il delitto lo avete messo nel bilancio di famiglia!». ${ }^{40}$

Alberto Saporito è dunque un affabulatore, un monologante, perché parla anche quando gli sembra inutile parlare, denunciando in un mondo di sordi, di "gente che parla cumme fosse muta", l'uccisione della "parola colorata», della comunicazione e della "stima reciproca» ("E vi sembra un assassinio da niente?»). ${ }^{41}$ D'altra parte il personaggio che rifiuta la parola, in questa commedia paradossale, è lo Zi' Nicola: il rapporto privilegiato fra «'o sparavierze», che sostituisce la parola con un codice alternativo, colorato e rumoristico, ed il nipote Alberto, sognatore e visionario, comunica il senso complessivo dello spettacolo del 1948. Così il protagonista si sdoppia, dal momento che si sono imbrogliate le lingue.

Si può ripercorrere la storia di questa commedia, dal primo manoscritto alle scene, attraverso copioni, testi editi e modificati dall'autore, e diverse edi-

38. E. De FILIPPO, Le voci di dentro, in Id., Cantata dei giorni dispari, cit., vol. I, atto III, p. 436.

39. Ivi, atto I, p. 397.

40. Ivi, atto III, p. 436.

41. Ibidem. 
zioni dello spettacolo, fino ad arrivare all'ultima, interpretata dall'attore, quella del '76-'77. Ecco, in questa occasione è stata rilevata dagli osservatori una specie di simbiosi tra il linguaggio del silenzio di un personaggio e quello dell'affabulazione dell'altro. Scrive Tian: «Le visioni di Alberto e il codice di Zi' Nicola, sommati insieme, creano un clima da giudizio universale [...] Possiamo anticiparcelo e magari renderlo più umano se accettiamo di parlare un linguaggio diverso da quello abituale». ${ }^{42}$ Tanto più che l'attore offre del pezzo d'affabulazione finale, quello di Alberto, un'interpretazione che pare avvicinarsi progressivamente al suo codice del silenzio. Eduardo «non rompe l'aria con le parole, l'appanna col fiato», ${ }^{43}$ la sua voce si fa sempre più intima, riuscendo a descrivere un intero mondo con tratti sempre più brevi e sempre più intensi; incide e mostra il silenzio, quando vuole creare dramma e abisso col silenzio, fino a sembrare egli stesso la materializzazione di un sogno o di una visione.

Eppure così continua a rivelare nel suo modo sornione l'assurdità delle voci di fuori e la mostruosità delle voci di dentro di tutti noi... Essere attori e attori artisti è incarnare una tragedia, o, se si preferisce, denunciarla. «Questo compito ingrato —aggiunge Garboli- per ironia della sorte, lo assolvono più di tutti gli attori comici, quelli che fanno ridere!». ${ }^{4}$

42. R. TIAN, «Visioni e fuochi d'artificio per capir meglio la realtà», Il Messaggero, 21 gennaio 1977.

43. S. BolCHI, "Roma in fila per partecipare ai sogni di Eduardo De Filippo», Corriere della Sera, 8 febbraio 1977.

44. C. Garboli, Casa Cupiello, cit., p. 231. 